\title{
Lovastatin enhances the antitumoral and apoptotic activity of doxorubicin in murine tumor models
}

\author{
VIVIANA R. ROZADOS ${ }^{1}$, LUCILA I. HINRICHSEN ${ }^{1,2}$, M. MERCEDES BINDA ${ }^{3}$, SILVIA I. GERVASONI ${ }^{1}$, \\ PABLO MATAR $^{1}$, R. DANIEL BONFIL ${ }^{3,4}$ and O. GRACIELA SCHAROVSKY ${ }^{1,2}$ \\ ${ }^{1}$ Instituto de Genética Experimental, Facultad de Ciencias Médicas; ${ }^{2}$ Consejo de Investigaciones, \\ Universidad Nacional de Rosario; ${ }^{3}$ Laboratory of Fundación de Investigación del Cáncer, Center for \\ Pharmacological Studies (CEFYBO, CONICET), Buenos Aires, Argentina; ${ }^{4}$ Wayne State University \\ School of Medicine and Barbara Ann Karmanos Cancer Institute, Detroit, MI, USA
}

Received November 28, 2007; Accepted January 25, 2008

\begin{abstract}
Despite its effectiveness as an antineoplastic drug, doxorubicin (DOX) is usually associated with cardiotoxicity. Lovastatin (LOV), a hypolipidemic agent used in the clinic, has been demonstrated to have antitumoral and antimetastatic effects in murine models. Since the two agents arrest tumor cells in different phases of the cell cycle and induce apoptosis, the goal of this study was to examine the efficacy of a combination therapy with LOV and low doses of DOX, in an attempt to obtain an improved antitumoral effect devoid of toxicity, by using a rat B-cell lymphoma and a mouse mammary tumor. In the two models, the combined treatment showed a synergistic antitumoral effect, which is mainly ascribed to an increased apoptotic response elicited by a LOV/DOX combination than either agent alone. The therapeutic benefit demonstrated by the combination treatment is further emphasized by the lack of toxicity.
\end{abstract}

\section{Introduction}

The loss of the feedback inhibition mechanism that regulates cholesterol biosynthesis is an important feature of malignant transformation. It has been reported that cancer cells require higher amounts of cholesterol and cholesterol precursors than normal cells (1). Mevalonate, one such precursor, is a critical component of a complex biochemical pathway whose products, geranylgeranyl and farnesyl pyrophosphate, are needed for post-translational modification by the isoprenylation of

Correspondence to: Dr O. Graciela Scharovsky, Instituto de Genética Experimental, Facultad de Ciencias Médicas, UNR. Santa $\mathrm{Fe}$ 3100, (2000) Rosario, Argentina

E-mail:ogs@citynet.net.ar

Key words: lovastatin, doxorubicin, apoptosis proteins like Ras, Rho, Rac and lamin A and B (2). Isoprenylated Ras proteins associate with the cell membrane thus becoming capable of regulating the signal transduction pathways involved in the control of cell growth, differentiation and survival (3).

The regulation of the mevalonate synthesis is complex, involving multiple feedback mechanisms in which the end products of the pathway can regulate the activity of 3-hydroxyl3-methylglutaryl coenzyme A (HMG-CoA) reductase, the ratelimiting enzyme of this metabolic pathway (4). Lovastatin (LOV) is a specific competitive inhibitor of HMG-CoA reductase (5), extensively used in medical practice to treat hypercholesterolemia (6). LOV has also been shown to arrest tumor and normal cells in the $\mathrm{G}_{1}$ phase of the cell cycle, inducing a potent apoptotic response. Its antitumoral and antimetastatic effects have been demonstrated in experimental murine models (7-9).

Doxorubicin (DOX) is an anthracycline antibiotic isolated from the fungus Streptomyces peucetius (10). It induces the formation of covalent topoisomerase-DNA complexes (11), cell cycle arrest in the $G_{2}$ phase and apoptosis (12). DOX has been in clinical practice since the 1960 s, and is considered one of the most effective drugs in the treatment of lymphomas, breast adenocarcinomas and other malignant tumors (13). However, it has been associated with the development of acute and chronic cardiac toxicity (14).

The aforementioned information, as well as the knowledge that LOV and DOX are able to stop the cell cycle in the different phases $\left(G_{1}\right.$ and $G_{2}$, respectively) $(12,15)$, prompted us to study the feasibility of a combined therapeutic intervention in which lower doses of DOX could be used. This therapeutic scheme would likely decrease the toxic effects of DOX, while improving the effectiveness of the therapeutic response. Herein, we examined the antitumoral effect of LOV and the non-toxic doses of DOX in the two animal tumor models of the rat lymphoma L-TACB and the mouse mammary adenocarcinoma M-406. We investigated the effect of the combined treatment by analyzing cell viability in vitro, studying tumor growth and apoptosis in vivo, and evaluating the variations of body weight as a general indicator of animal health status. 


\section{Materials and methods}

Animals. Ten to 12-week-old female inbred IIM e/Fm (from here on $e$ ) rats (16) and 6- to 8-week-old inbred $\mathrm{CBi}$ female mice (17) were used throughout and obtained from the Facultad de Ciencias Médicas, Universidad Nacional de Rosario, breeding facilities. Animals were fed a commercial chow and water ad libitum and were maintained in a $12 \mathrm{~h}$ light/dark cycle. All the experiments were done during the first half of the light cycle and according to animal care standards of the institution, which complies with the guidelines issued by the Canadian Council on Animal Care (Ontario, Canada, 1993).

Drugs. The inactive lactone form of LOV (GADOR, Argentina) was converted to its active form by dissolving $480 \mathrm{mg}$ in $12.5 \mathrm{ml}$ of $96 \%$ ethanol and $18 \mathrm{ml}$ of $0.1 \mathrm{M} \mathrm{NaOH}$ at $50^{\circ} \mathrm{C}$ for $2 \mathrm{~h}$. The solution was neutralized with $0.1 \mathrm{M} \mathrm{HCl}$ to $\mathrm{pH} 7.0$ and brought to a final volume of $60 \mathrm{ml}$ with distilled water. Aliquots of this stock solution $(20 \mathrm{mM})$ were stored frozen at $-20^{\circ} \mathrm{C}$ until used. DOX (Filaxis S.A., Argentina) was diluted to $1 \mathrm{mg} / \mathrm{ml}$ in saline solution before injection.

Tumors. L-TACB is a poorly-differentiated B-cell lymphoma that arose spontaneously in an inbred $e$ rat (18). It is maintained by serial subcutaneous trocar implantation of $1 \mathrm{~mm}^{3}$ tumor fragments $\left(\sim 10^{6}\right.$ cells $)$ in syngeneic rats. M-406 is a type B semi-differentiated mammary adenocarcinoma according to Squartini's classification (19) which occurred spontaneously in an inbred $\mathrm{CBi}$ female mouse (20). It is maintained in vivo in syngeneic mice.

Chemosensitivity assay. L-TACB and M-406 cells obtained by mechanical disruption of subcutaneous primary tumors excised 14 days after challenge, were suspended in RPMI1640 culture medium (Sigma Chemical Co., St. Louis, MO) and supplemented with $10 \%$ fetal bovine serum (FBS, Natocor, Córdoba, Argentina). Cell suspensions with $>95 \%$ viability were seeded in 96-well flat-bottomed microplates (Corning Costar) at $1 \times 10^{5}$ cells per well in a $200 \mu 1$ complete culture medium. Four groups were established to test the effect of the different treatments on cell viability: 1) control, without treatment; 2) LOV, treated with $20 \mathrm{mM} \mathrm{LOV}$; 3) DOX, treated with $1 \mathrm{mg} / \mathrm{ml}$ (L-TACB) or with $8 \mathrm{mg} / \mathrm{ml}$ (M-406) and 4) $\mathrm{LOV}+\mathrm{DOX}$, treated with $20 \mathrm{mM} \mathrm{LOV}$ plus 1 or $8 \mathrm{mg} / \mathrm{ml}$ DOX (L-TACB and M-406 cells, respectively). After $24 \mathrm{~h}$ incubation at $37^{\circ} \mathrm{C}$ in a $5 \% \mathrm{CO}_{2}$ humidified atmosphere, a trypan blue dye exclusion test was performed and cell viability was determined. The determinations were done in quadruplicate.

In vivo experimental models. Rats and mice were injected subcutaneously in the right flank with L-TACB and M-406 fragments $\left(\sim 10^{6}\right.$ cells $)$, respectively, on day 0 . The animals were distributed in four groups and injected by i.p. route. For M-406: 1) saline (control group); 2) LOV (25 mg/kg of body weight) three times a week from day 5 to $21 ; 3)$ DOX $(1 \mathrm{mg} / \mathrm{kg}$ of body weight), twice a week from day 5-21; 4) LOV + DOX as in groups 2 and 3. For L-TACB: 1) saline (control group); 2) LOV (25 mg/kg of body weight) daily from day 0 to $4 ; 3$ )
DOX $(0.5 \mathrm{mg} / \mathrm{kg})$ on day 10 and 4) LOV + DOX as in groups 2 and 3 . The treatment schedule for each tumor model was chosen based on several regimens tested before with different DOX and LOV doses or different times for initiation of the therapy to achieve a better antitumoral effect with a lesser toxicity (data not shown). Tumor diameters were measured twice a week with a caliper to calculate tumor volume using the formula $\mathrm{v}=0.4\left(\mathrm{ab}^{2}\right)$, where $\mathrm{v}$ is the volume $\left(\mathrm{cm}^{3}\right)$; $\mathrm{a}$, largest diameter $(\mathrm{cm})$ and $\mathrm{b}$, smallest diameter $(\mathrm{cm})$. Body weights were recorded twice a week throughout the experiments in addition to monitoring the general health status for signs and symptoms of toxicity. The animals were sacrificed on days 21 (L-TACB) or 26 (M-406), and their tumors resected and processed for histological examination. The end point of each experiment was set to the day in which the tumors of the control animals reached the maximum permitted volume.

Immunohistochemistry. Excised tumors were fixed in $10 \%$ buffered formalin and embedded in paraffin. After the deparaffinization and re-hydration of the sections, endogenous peroxidase was quenched using $3 \%$ hydrogen peroxide for $5 \mathrm{~min}$. Non-specific binding was blocked with normal goat serum. Slides were washed for 5 min with tris-buffered saline solution containing Tween-20 at pH 7.6 and incubated with: a) rabbit anti-Bcl-2 antibody (Santa Cruz Biotechnology Inc., Santa Cruz, CA) (1:50) followed by incubation with horseradish peroxidase-conjugated goat anti-rabbit IgG antibody (Dako) (1:150) or, b) mouse anti-Bax antibody (Santa Cruz Biotechnology) (1:50) followed by incubation with Vectastain ${ }^{\circledR}$ Elite ABC (Mouse IgG, Vector Laboratories, Burlingame, CA). Immunostainings were developed with 3,3'-diaminobenzidine (Sigma) and the sections were lightly counterstained with methyl green. Negative controls were carried out by omitting the primary antibody. Slides were washed in tap water, dehydrated and mounted with glass coverslips. The intensity of Bcl-2 and Bax staining in tumor lesions was assessed at high power $(\mathrm{x} 400)$ and graded on a scale of 0 to +4 by two independent investigators blinded to sample identity, with 0 indicating undetectable staining and +4 the strongest staining.

TUNEL assay. The level of intratumoral apoptosis was determined by the terminal deoxynucleotidyl transferasemediated deoxyuridine triphosphate-peroxidase nick end labeling (TUNEL) technique. Briefly, apoptotic cells were identified in the sections using the TUNEL method (ApopTag ${ }^{\circledR}$ Peroxidase in situ apoptosis detection kit for immunoperoxidase staining, Intergen Company, Purchase, NY) following the manufacturer's instructions. Apoptotic cells were counted in 10 microscopic fields, chosen at random, at a magnification of $\mathrm{x} 100$.

Statistical analysis. Data were statistically analyzed using Student's t-test. Values of $\mathrm{P}<0.05$ were considered statistically significant.

\section{Results}

The effect of $L O V$ and DOX on tumor cell viability. The in vitro cytotoxic effect of treatments with LOV, DOX or the combin- 

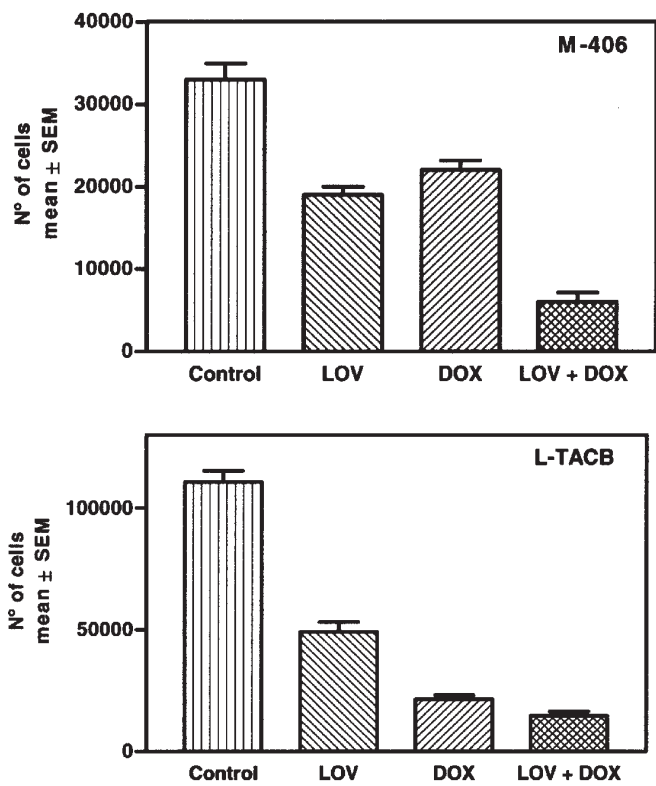

Figure 1. The in vitro effect of LOV, DOX and their combination on tumor cell viability. The data are mean \pm SEM of viable cells as determined by the trypan blue exclusion test. M-406: control vs. LOV + DOX, LOV vs. $\mathrm{LOV}+\mathrm{DOX}$, DOX vs. LOV + DOX, $\mathrm{P}<0.001$, control vs. DOX $\mathrm{P}<0.01$. L-TACB: control vs. LOV, control vs. DOX, control vs. LOV + DOX, LOV vs. $\mathrm{LOV}+\mathrm{DOX}, \mathrm{P}<0.001, \mathrm{DOX}$ vs. $\mathrm{LOV}+\mathrm{DOX}, \mathrm{P}<0.01$. The statistical significance of the differences between the groups was assessed by Student's t-test. Each experiment was performed in quadruplicate.
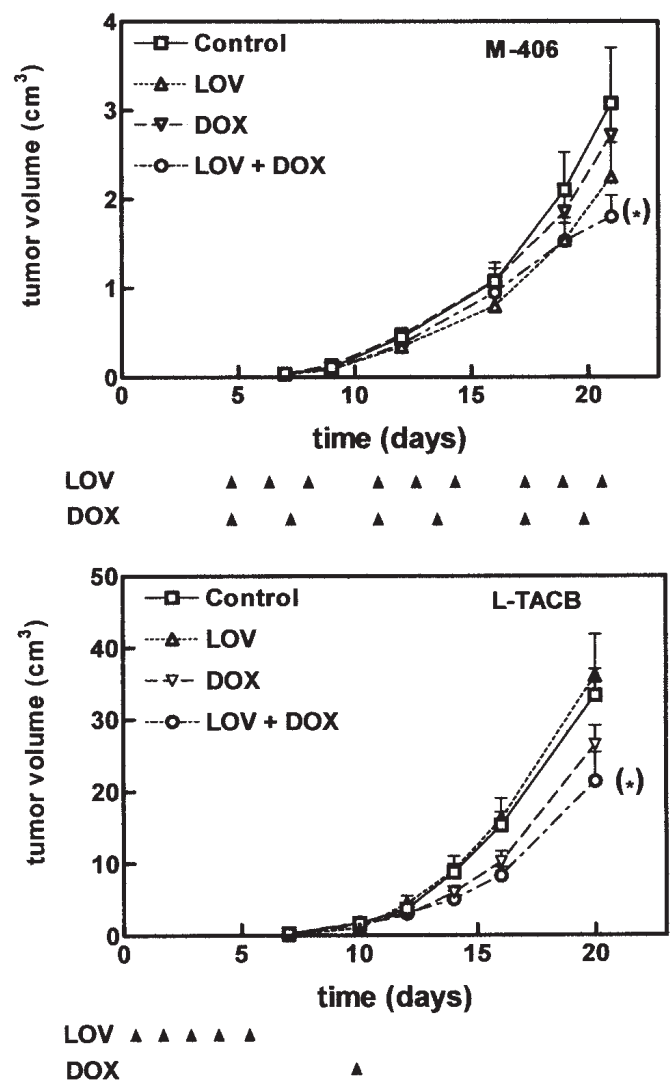

Figure 2. The effect of the treatment with LOV, DOX or LOV + DOX on the tumor growth. The data for each time-point are mean \pm SEM (M-406, $\mathrm{n}=12$ and L-TACB, $\mathrm{n}=9$ per treatment group). The arrowheads indicate specific times at which LOV or DOX were administered. M-406 (day 21) and L-TACB (day 20): control vs. LOV + DOX, P<0.05 (Student's t-test). ation of the two drugs was studied on M-406 and L-TACB cells by measuring cell viability. Fig. 1 shows the effect of the different treatments on tumor cell viability after $24 \mathrm{~h}$ culture. Treatment with LOV or DOX alone induced a significant decrease in cell viability as compared to the control. Notably, the combined treatment (LOV + DOX) produced a synergistic effect on tumor cell viability, which was statistically lower than that obtained with each single drug in the two tumor models.

The effect of LOV and DOX treatment on in vivo tumor growth. In vivo studies were performed to examine whether our in vitro findings had potential therapeutic significance. The doses of LOV and DOX used in combination, were chosen among several tested (results not shown), and were those that resulted in a significant therapeutic response with negligible toxic effects. The schedules finally chosen were LOV $(25 \mathrm{mg} / \mathrm{kg})$ three times a week from day 5 to 21 plus DOX (1 mg/kg), twice a week from day 5 to 21 for the M-406 tumor model and LOV $(25 \mathrm{mg} / \mathrm{kg})$ daily from day 0 to 4 , plus DOX $(0.5 \mathrm{mg} / \mathrm{kg})$ on day 10 for the L-TACB tumor model. At the doses used, neither LOV nor DOX exerted a significant antitumoral effect on either of the tumor models as single drugs, as compared to the control groups. Conversely, the combined treatment with LOV and DOX delayed the tumor growth in the two tumor systems significantly. At the end of the experiment, the combined treatment induced a reduction in tumor size of 42 and $35 \%$ for M-406 and L-TACB, respectively, when compared to the controls $(\mathrm{p}<0.05)$ (Fig. 2).

In order to assess the toxicity of the different treatments, we recorded body weight throughout the experiment, since its variation is a good indicator of general health status. At the end of the experiment, the mean increase in body weight of the animals in the control groups (11.7 and $11.3 \%$ for M-406 and L-TACB, respectively) was higher than those in the treated groups (range of increase: $3.9-8.6 \%$, respectively). This difference in weight gain may be ascribed in part to significantly larger tumor masses in the control groups. Nevertheless, it should be pointed out that the aim of this analysis was not to compare differences in body weights among the different treatment groups, but to evaluate whether a reduction in body weight that happened within each group could be used as an indicator of toxicity. In this respect, it is important to point out that none of the treated animals exhibited weight loss during the experiment. The evolution of the weight gain during the period studied is plotted in Fig. 3 as the percentage of its value at the beginning of the experiment for each treatment group. Moreover, no alterations in motor activity, fur quality, food intake, reflexes and breathing were observed, this being indicative of a treatment with low or no toxicity. Also, no drug-related deaths were observed.

The effect of LOV and DOX on in vivo tumor apoptosis. The induction of apoptosis by DOX and LOV, either alone or in combination, was assessed by estimating the number of TUNEL-positive cells in the two tumor models at the end of the experiment (Fig. 4). Only M-406 and L-TACB tumors harvested from animals treated with LOV + DOX showed a significant increase in the number of apoptotic cells when compared to tumors in the control groups $(\mathrm{p}<0.05)$. The 

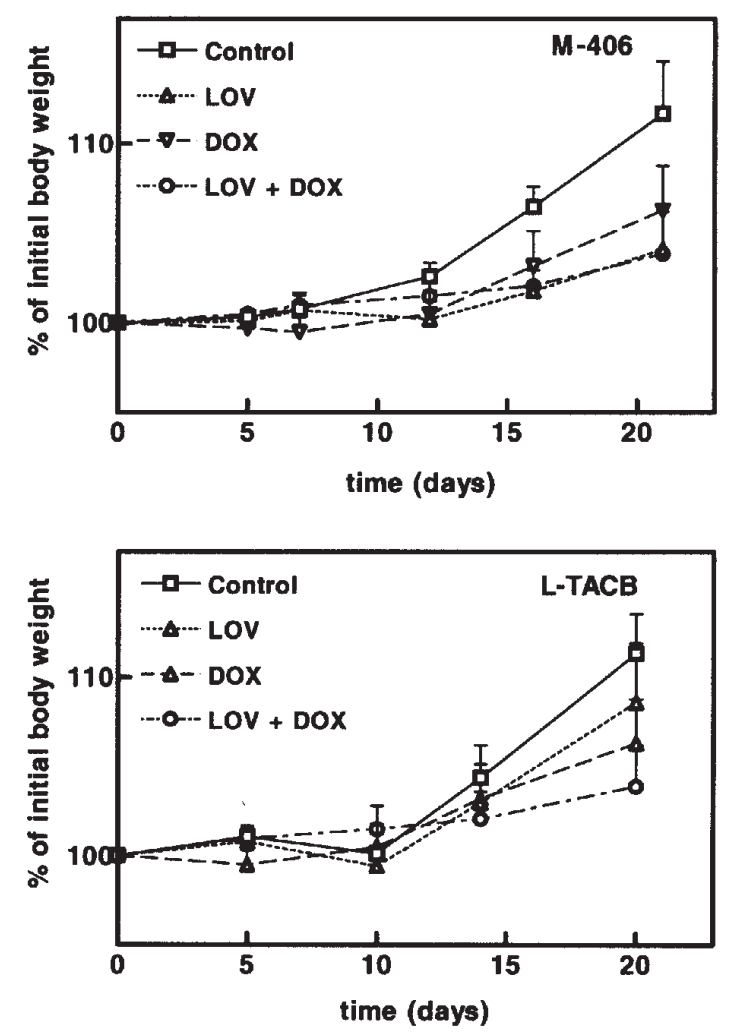

Figure 3. Evolution of body weight. The data are plotted as a percentage of body weight at the beginning of the experiment; each time-point is mean \pm SEM (M-406, n=12 and L-TACB, n=9 per treatment group), Student's t-test.
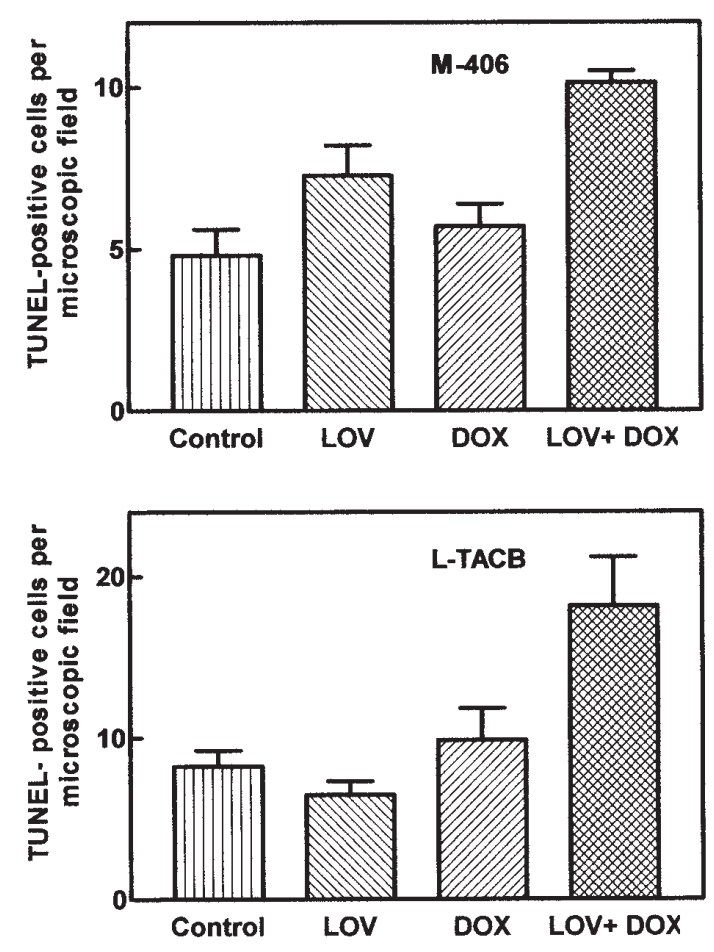

Figure 4. Apoptosis of M-406 and L-TACB cells treated with LOV, DOX or LOV + DOX, as determined by TUNEL staining. The data are mean \pm SEM of TUNEL-positive cells per microscopic field (magnification, x100). M-406: control vs. LOV + DOX, LOV vs. LOV + DOX, DOX vs. LOV + DOX, $\mathrm{P}<0.05$. L-TACB: control vs. LOV + DOX, DOX vs. LOV + DOX, $\mathrm{P}<0.05$, LOV vs. LOV + DOX, P<0.01 (Student's t-test).

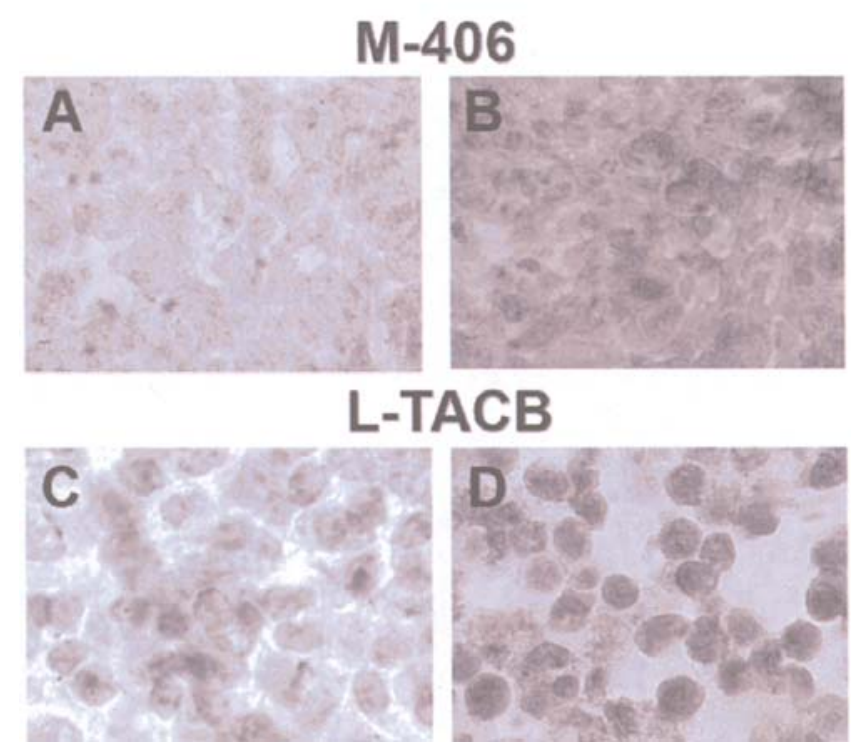

Figure 5. The immunohistochemical analysis of Bax expression in sections of M-406 and L-TACB tumors. M-406: (A) control, (B) LOV + DOX and L-TACB: (C) control, (D) LOV + DOX (magnification, $x 400$ ). Tissue samples were obtained after 21 and 20 days of tumor challenge with M-406 or L-TACB, respectively. The intensity of Bax expression was higher in the tumors of the animals treated with LOV + DOX (B and D) when compared to tumors of the non-treated animals ( $\mathrm{A}$ and $\mathrm{C}$ ), respectively.

expression of the anti- and pro-apoptotic molecules Bcl-2 and Bax, respectively, was also assessed immunohistochemically in the two tumors under the different treatments. No significant modification in the expression of $\mathrm{Bcl}-2$ was evident in the tumors from the combined treatment as well as the single treatment groups with respect to the control tumors (data not shown). On the contrary, the expression of Bax was higher in the LOV + DOX treated tumors than in the control tumors (Fig. 5). Bax expression in tumors treated either with LOV or DOX, as single drugs, was similar to that observed in the controls or showed intermediate levels of expression. The intensity of Bax expression in M-406 was 0 (undetectable) in tumors from the control and DOX single treatment groups, +2 in LOV-treated tumors and +3 in the combined treatment group LOV + DOX. In L-TACB tumors, Bax expression was +2 in control samples, +3 in the samples from the two single treatments (LOV or DOX) and +4 in the LOV + DOX combined treatment samples.

\section{Discussion}

One of the current goals in cancer therapy is to reduce the development of side effects of chemotherapy and radiotherapy while retaining their antitumoral capacity. As we have already demonstrated in the sarcoma and lymphoma rat tumor models $(8,9)$, LOV has antitumoral and/or antimetastatic activity, with no observable toxicity at the dose and schedule used. Other authors have also shown the antitumoral effect of LOV either alone $(7,21)$ or in combination with different drugs $(22-24)$.

Based on the above-mentioned results, and taking into account the known ability of LOV and DOX to stop the cell cycle in different phases $(12,15)$, we investigated whether the 
combination of low doses of DOX with LOV would result in high therapeutic efficacy with low or no toxic side-effects. Previous results showing that LOV can reduce the cardiotoxicity produced by DOX (25), as well as those indicating that the therapeutic efficacy of DOX and its derivatives is improved when combined with other drugs (26), also supported our selection of DOX for the combined treatments in the mammary adenocarcinoma and lymphoma tumor models used herein.

We found that low doses of LOV or DOX, when used as single agents, decrease tumor cell viability in the in vitro assays. Notably, the use of the two drugs in combination induced a significant increase in cytotoxicity with respect to each single treatment. These results are in agreement with those obtained by Feleszko et al (27) in sarcoma, colon and lung carcinoma tumor-models. The combination of LOV with cisplatin (23), sulindac (28) or paclitaxel (29) also yielded similar results. Riganti et al recently demonstrated that statins revert the resistance of a human malignant mesothelioma cell line to DOX (30). Contrasting with the results mentioned above, Bardeleben et al (31) working with CHO-K1, HepG2 and Jurkat cells, found that pretreatment with LOV rendered them resistant to DOX. Such a disparity between the results from several laboratories, including our own, is the consequence of differences in the experimental conditions used, the cell type assayed and/or the presence or not of a mutated ras gene. Certainly, contradictory results such as those described deserve further studies, in vitro and in vivo.

The enhanced cytotoxic effect of the combined treatment observed in the in vitro experiments for the two tumors led us to study this drug combination in an in vivo setting. The simultaneous administration of LOV and DOX to M-406bearing mice resulted in antitumoral activity not achieved by DOX when given alone. While the rate of tumor growth in animals treated with either agent did not differ from the controls in the period studied, a significant decrease in tumor volume was demonstrated in the mice receiving the combined treatment. The outcome in the lymphoma L-TACB model was similar to that observed in the mammary adenocarcinoma M-406. Tumor volumes on days 20 (L-TACB) or 21 (M-406) were smaller in LOV + DOX treated animals when compared to each control group, indicating a delay in tumor growth. These in vivo results are in agreement with those obtained by Feleszko et al with melanoma cell lines (32). The treatment schedules employed [administration of LOV and DOX in a sequential (M-406) or simultaneous (L-TACB) manner] were the best of several tested, resulting in a significantly diminished growth rate when compared to the controls. It cannot be concluded from our experiments whether LOV or DOX modified the pharmacokinetic properties of each other. However, at least for the L-TACB tumor model, we can assume that such an alteration did not occur, because the last injection of LOV, a drug with a short half life, was given 6 days before beginning the administration of DOX.

Body weight, a variable currently used as an indicator of health status, did not decrease in any of the groups during the period studied, for either of the tumors used. On the contrary, a steady increase of body weight was observed throughout the experiments. This result, together with the lack of clinical signs of drug toxicity such as anorexia, cachexia, raffled fur or skin tenting, supports our hypothesis that the combined treatment is able to preserve the antitumoral efficacy of higher drug doses while decreasing its toxicity.

It has been demonstrated that statins, alone or in combination with other drugs, are able to induce apoptosis in different tumor cells $(33,34)$. Since the enhancement of apoptosis may be a mechanism implicated in the in vivo antitumoral effect, the presence of apoptotic cells in tumor tissue was evaluated by the TUNEL assay. The results obtained are in agreement with those derived from the in vitro and in vivo experiments. The combined treatments in M-406 and L-TACB resulted in tumors with a higher number of apoptotic cells than in all the other experimental groups. In the two tumor models, the level of apoptosis determined in the tumors obtained from animals treated with a single drug did not differ from that of the controls. The amount of apoptosis induced by LOV or DOX alone was not enough to reach the threshold needed to evince an effect on tumor development. On the contrary, the combined treatment affected tumor growth, suggesting that LOV was able to increase the levels of apoptosis induced by a low dose of DOX.

There are several molecules involved in the activation and inhibition of apoptosis, Bax and Bcl-2 being two of the most conspicuous. The immunohistochemical study of these molecules in the tumoral tissue, used to assess their possible modulation by the combined treatment, showed that Bcl-2 expression was not significantly affected, while the expression of Bax was evidently up-regulated in the lymphoma and the adenocarcinoma tumors. Therefore, one of the molecules involved in enhanced apoptosis as produced by the combined treatment, would be Bax. However, additional molecules regulating apoptosis other than Bax should not be excluded as responsible of the effect due to the combined treatment and, therefore, this deserves future research.

Other mechanisms may also be responsible for the increased apoptotic level achieved with the administration of the combined treatment. Of these, the effect of LOV on the p21ras protein function is particularly attractive, since the activated p21ras protein may alter the development of apoptosis through the PI3K pathway (35). In addition, the pharmacological action of LOV on cancer cells is observed even in those cells with non-mutated ras oncogenes (36).

The inhibition of HMG-CoA reductase by LOV implies a decreased synthesis of isoprenyl groups like farnesyl. Since farnesylation is necessary to anchor p21ras to the cell membrane and enable this protein to function as a signal transducer (3), LOV treatment would result in the impairment of the p21 function. In agreement with this assumption, we previously found that treatment of L-TACB-bearing rats with LOV diminished the levels of p21ras tethered to the membrane in tumors with respect to non-treated animals significantly (37). These results suggest that, under our experimental conditions, LOV inhibits the membrane association of p21ras in L-TACB cells. Thus, the decrease of in vitro cell viability and the enhancement of apoptosis in vivo, observed when LOV was associated with DOX, could be partially explained by the known involvement of the $\mathrm{p} 21$ ras protein in proliferation signaling $(38,39)$, as well as by the inhibition of apoptosis $(40,41)$, which would add to the known effect of DOX on the two parameters (12). 
It can be argued that the direct effect of LOV on its molecular target is reversible after its withdrawal. Nevertheless, our past and present experimental results show that the indirect effect(s) of the drug is (are), at least in part, nonreversible. We previously reported that an identical schedule of LOV administration as in the present experiments led to the inhibition of metastasis development in the L-TACB tumormodel (9). In general, it is accepted that the development of the invasive and metastatic phenotypes is not an early event in tumor progression. For this reason, it is reasonable to consider that the treatment with LOV on days 0 to 4 would affect events that take place later on. Moreover, the inhibition of the p21ras membrane anchorage on L-TACB cells determined four days after LOV withdrawal, showed a decrease of the p21ras protein in the membrane fraction, indicating that the indirect effect of LOV was still occurring (37).

In summary, we have demonstrated the feasibility of treating different tumor types with a combination of low doses of DOX and LOV and obtaining a delay in tumor growth without signs of toxic side effects. These results provide additional information on the role of statins as enhancers of the cytotoxic effect of chemotherapeutic drugs. Moreover, the therapeutic use of statins in combination with low-dose standard chemotherapy could fulfill the ever-present aim of cancer treatment to achieve an optimal antitumoral effect with minimal toxicity. Since LOV pharmacodynamics and pharmacokinetics are well characterized, it is foreseeable that it could be incorporated in combination with cytotoxic drugs into phase I clinical trials in the near future.

\section{Acknowledgements}

We thank the collaboration of Stella Roma in the evaluation of the immunohistochemistry images and GADOR, Argentina, for providing the lovastatin employed herein.

\section{References}

1. Buchwald H: Cholesterol inhibition, cancer, and chemotherapy. Lancet 339: 1154-1156, 1992.

2. Khosravi-Far R, Cox AD, Kato K and Der CJ: Protein prenylation: key to ras function and cancer intervention? Cell Growth Differ 3: 461-469, 1992.

3. Scharovsky OG, Rozados VR, Gervasoni SI and Matar P: Inhibition of ras oncogene: a novel approach to antineoplastic therapy. J Biomed Sci 7: 292-298, 2000.

4. Endo A and Kuroda M: Citrinin, an inhibitor of cholesterol synthesis. J Antibiot 29: 841-843, 1976.

5. Brown MS, Faust JR and Goldstein JL: Induction of 3-hydroxy3-methylglutaryl coenzyme A reductase activity in human fibroblasts incubated with compactin (ML-236B), a competitive inhibitor of the reductase. J Biol Chem 253: 1121-1128, 1978.

6. Alberts A, MacDonald J, Till A and Tobert J: Lovastatin. Cardiovasc Drug Rev 7: 89-109, 1989.

7. Maltese WA, Defendini R, Green RA, Sheridan KM and Donley DK: Suppression of murine neuroblastoma growth in vivo by mevinolin, a competitive inhibitor of 3-hydroxy-3-methylglutaryl-coenzyme A reductase. J Clin Invest 76: 1748-1754, 1985.

8. Matar P, Rozados VR, Roggero EA and Scharovsky OG: Lovastatin inhibits tumor growth and metastasis development of a rat fibrosarcoma. Cancer Biother Radiopharm 13: 387-393, 1998.

9. Matar P, Rozados VR, Binda MM, Roggero EA, Bonfil RD and Scharovsky OG: Inhibitory effect of Lovastatin on spontaneous metastases derived from a rat lymphoma. Clin Exp Metastasis 17: 19-25, 1999.

10. Marco A and Arcamone F: DNA complexing antibiotics: daunomycin, adriamycin and their derivatives. Arzneimittelforschung 25: 368-374, 1975 .
11. Corbett AH and Osheroff N: When good enzymes go bad: conversion of topoisomerase II to a cellular toxin by antineoplastic drugs. Chem Res Toxicol 6: 585-597, 1993.

12. Mansilla S, Pina B and Portugal J: Daunorubicin-induced variations in gene transcription: commitment to proliferation arrest, senescence and apoptosis. Biochem J 372: 703-711, 2003.

13. DeVita Jr VT, Hellman S and Rosenberg SA: Cancer: Principles and Practice of Oncology. 5th edition, Lippincott-Raven, New York, 2000.

14. Allen A: The cardiotoxicity of chemotherapeutic drugs. Semin Oncol 19: 529-542, 1992.

15. Keyomarsi K, Sandoval L, Band V and Pardee AB: Synchronization of tumor and normal cells from $\mathrm{G} 1$ to multiple cell cycles by lovastatin. Cancer Res 51: 3602-3609, 1991.

16. Calderari S, Font MT, Garrocq O, Martinez S, Morini JC, Puche R, et al: The inbred IIM/Fm stock. Rat News Lett 25: 28-29, 1991.

17. Di Masso RJ, Abdala S, Sanchez SM and Font MT: Respuesta a la selección divergente por conformación corporal en el ratón. Mendeliana 9: 79-92, 1991.

18. Celoria GC, Hinrichsen LI and Font MT: Tumor behavior of lymphoma TACB in rats resistant or susceptible to Sarcoma E100. Comp Biol 5: 73-83, 1986.

19. Squartini $\mathrm{F}$ and Pingitore R: Tumours of the mammary gland. IARC Sci Publ 111: 47-100, 1994.

20. Rozados V: Análisis de la actividad antitumoral y antimetastásica del Lovastatin en modelos tumorales murinos. PhD Thesis, Universidad Nacional de Rosario, Rosario, Argentina, 2004.

21. Jani JP, Specht S, Stemmler N, Blanock K, Singh SV, Gupta V, et al: Metastasis of B16F10 mouse melanoma inhibited by lovastatin, an inhibitor of cholesterol biosynthesis. Invasion Metastasis 13: 314-324, 1993.

22. Soma MR, Baetta R, De Renzis MR, Mazzini G, Davegna C, Magrassi L, et al: In vivo enhanced antitumor activity of carmustine [N,N'-bis(2-chloroethyl)-N-nitrosourea] by simvastatin. Cancer Res 55: 597-602, 1995.

23. Feleszko W, Zagozdzon R, Golab J and Jakobisiak M: Potentiated antitumour effects of cisplatin and lovastatin against MmB16 melanoma in mice. Eur J Cancer 34: 406-411, 1998.

24. Feleszko W, Balkowiec EZ, Sieberth E, Marczak M, Dabrowska A, Giermasz A, et al: Lovastatin and tumor necrosis factor-alpha exhibit potentiated antitumor effects against Haras-transformed murine tumor via inhibition of tumor-induced angiogenesis. Int J Cancer 81: 560-567, 1999.

25. Iliskovic $\mathrm{N}$ and Singal PK: Lipid lowering: an important factor in preventing adriamycin-induced heart failure. Am J Pathol 150: 727-734, 1997.

26. Rosenthal MA, Green M, Wiernik P, Borden EC, Marsh JC and Haller DG: Epirubicin has modest single-agent activity in head and neck cancer but limited activity in metastatic melanoma and colorectal cancer: phase II studies by the Eastern Cooperative Oncology Group. Am J Clin Oncol 21: 509-512, 1998.

27. Feleszko W, Mlynarczuk I, Balkowiec-Iskra EZ, Czajka A, Switaj T, Stoklosa T, et al: Lovastatin potentiates antitumor activity and attenuates cardiotoxicity of doxorubicin in three tumor models in mice. Clin Cancer Res 6: 2044-2052, 2000.

28. Agarwal B, Rao CV, Bhendwal S, Ramey WR, Shirin H, Reddy BS, et al: Lovastatin augments sulindac-induced apoptosis in colon cancer cells and potentiates chemopreventive effects of sulindac. Gastroenterology 117: 838-847, 1999.

29. Holstein SA and Hohl RJ: Synergistic interaction of lovastatin and paclitaxel in human cancer cells. Mol Cancer Ther 1: 141-149, 2001.

30. Riganti C, Orecchia S, Pescarmona G, Betta PG, Ghigo D and Bosia A: Statins revert doxorubicin resistance via nitric oxide in malignant mesothelioma. Int J Cancer 119: 17-27, 2006.

31. Bardeleben RV, Dunkern T, Kaina B and Fritz G: The HMGCoA reductase inhibitor lovastatin protects cells from the antineoplastic drugs doxorubicin and etoposide. Int J Mol Med 10: 473-479, 2002.

32. Feleszko W, Mlynarczuk I, Olszewska D, Jalili A, Grzela T, Lasek W, et al: Lovastatin potentiates antitumor activity of doxorubicin in murine melanoma via an apoptosis-dependent mechanism. Int J Cancer 100: 111-118, 2002.

33. Dmoszynska A, Podhorecka M, Klimek P and Grzasko N: Lovastatin and thalidomide have a combined effect on the rate of multiple myeloma cell apoptosis in short term cell cultures. Eur J Clin Pharmacol 62: 325-329, 2006.

34. Cafforio P, Dammacco F, Gernone A and Silvestris F: Statins activate the mitochondrial pathway of apoptosis in human lymphoblasts and myeloma cells. Carcinogenesis 26: 883-891, 2005. 
35. Hernandez-Alcoceba R, del Peso L and Lacal JC: The Ras family of GTPases in cancer cell invasion. Cell Mol Life Sci 57: 65-76, 2000.

36. Sumi S, Beauchamp RD, Townsend CM Jr, Pour PM, Ishizuka J and Thompson JC: Lovastatin inhibits pancreatic cancer growth regardless of RAS mutation. Pancreas 9: 657-661, 1994.

37. Rozados VR, Binda MM, Matar P, Gervasoni SI, Bonfil RD and Scharovsky OG: Inhibition of $\mathrm{p} 21^{\text {ras }}$ membrane anchorage by Lovastatin treatment of a rat lymphoma. Biocell 24: 171, 2000.

38. Sun J, Qian Y, Hamilton AD and Sebti SM: Ras CAAX peptidomimetic FTI 276 selectively blocks tumor growth in nude mice of a human lung carcinoma with K-Ras mutation and p53 deletion. Cancer Res 55: 4243-4247, 1995.
39. Donaldson MJ, Skoumas V, Watson M, Ashworth PA, Ryder H, Moore M, et al: XR3054, structurally related to limonene, is a novel inhibitor of farnesyl protein transferase. Eur J Cancer 35: 1014-1019, 1999.

40. Kauffmann-Zeh A, Rodriguez-Viciana P, Ulrich E, Gilbert C, Coffer P, Downward J, et al: Suppression of c-Myc-induced apoptosis by Ras signalling through PI(3)K and PKB. Nature 385: 544-548, 1997

41. Khwaja A, Rodriguez-Viciana P, Wennstrom S, Warne PH and Downward J: Matrix adhesion and Ras transformation both activate a phosphoinositide 3-OH kinase and protein kinase B/Akt cellular survival pathway. EMBO J 16: 2783-2793, 1997. 